PROCEEDINGS OF THE

AMERICAN MATHEMATICAL SOCIETY

Volume 135, Number 7, July 2007, Pages 2007-2009

S 0002-9939(07)08787-4

Article electronically published on February 6, 2007

\title{
EXTENSIONS OF ORTHOSYMMETRIC LATTICE BIMORPHISMS REVISITED
}

\author{
KARIM BOULABIAR
}

(Communicated by Joseph A. Ball)

\begin{abstract}
This note furnishes an example illustrating the following two facts. On the one hand, there exist Archimedean Riesz spaces $E$ and $F$ with $F$ Dedekind-complete and an orthosymmetric lattice bimorphism $\Psi: E \times E \rightarrow F$ with lattice bimorphism extension $\Psi^{\delta}: E^{\delta} \times E^{\delta} \rightarrow F$ which is not orthosymmetric, where $E^{\delta}$ denotes the Dedekind-completion of $E$. On the other hand, there is an associative $d$-multiplication $*$ in the same Archimedean Riesz space $E$ which extends to a $d$-multiplication $*^{\delta}$ in $E^{\delta}$ which is not associative. The existence of such an example provides counterexamples to assertions in Toumi, 2005.
\end{abstract}

We use the books [1] by Aliprantis and Burkinshaw and 3 by Gillman and Jerison as a starting point, and we refer the reader to these monographs for terminology, notation, and facts not explained or proved below.

Throughout this note, $E$ and $F$ stand for Archimedean Riesz spaces and $E^{\delta}$ indicates the Dedekind-completion of $E$. A bilinear map $\Psi: E \times E \rightarrow F$ is said to be positive if $\Psi(f, g) \in F^{+}$for all $f, g \in E^{+}$. Moreover, the bilinear map $\Psi$ is called a lattice bimorphism if the equality $|\Psi(f, g)|=\Psi(|f|,|g|)$ holds in $F$ for all $f, g \in E$. Furthermore, we say that the bilinear map $\Psi$ is orthosymmetric after Buskes and van Rooij [2] if $\Psi(f, g)=0$ in $F$ whenever $f \wedge g=0$ in $E$. On the other hand, Kudláček [4 has called a multiplication $*$ in $E$ a $d$-multiplication if the bilinear map $*: E \times E \rightarrow E$ defined by

$$
*(f, g)=f * g \quad \text { for all } f, g \in E
$$

is a lattice bimorphism.

Our purpose here is to provide an example which illustrates the following two statements:

1.1. There exist two Archimedean Riesz spaces $E$ and $F$ with $F$ Dedekindcomplete and an orthosymmetric lattice bimorphism $\Psi: E \times E \rightarrow F$ with lattice bimorphism extension $\Psi^{\delta}: E^{\delta} \times E^{\delta} \rightarrow F$ which is not orthosymmetric.

Received by the editors March 8, 2006 and, in revised form, April 19, 2006.

2000 Mathematics Subject Classification. Primary 06F25, 47B65.

Key words and phrases. Extension, Dedekind-completion, $d$-multiplication, lattice bimorphism, orthosymmetric, Riesz space.

The author would like to thank the referee for his helpful suggestions and comments which considerably improved preliminary versions of this work.

(C)2007 American Mathematical Society Reverts to public domain 28 years from publication 
1.2. There is an Archimedean Riesz space $E$ with an associative d-multiplication * which extends to a d-multiplication $*^{\delta}$ in $E^{\delta}$ which is not associative.

The existence of such an example provides counterexamples to some assertions in a recent paper of Toumi (see [5, Theorem 1] and [5, Theorem 2, Corollary 1, and Remark 1]).

As usual, the symbol $\mathbb{R}$ is used below to indicate the Dedekind-complete Riesz space of all real numbers. Moreover, $\mathbb{N}$ denotes the set of integers $\{1,2,3, \ldots\}$, and $C^{*}(\mathbb{N})$ is the set of all bounded real-valued continuous functions on $\mathbb{N}$. With the pointwise addition, scalar multiplication, product, and ordering, $C^{*}(\mathbb{N})$ is an algebra and a Dedekind-complete Riesz space. Furthermore, if $\beta \mathbb{N}$ denotes the Stone-Čech compactification of $\mathbb{N}$, then any function $f$ in $C^{*}(\mathbb{N})$ extends uniquely to a function $f^{\beta}$ in the algebra and Riesz space $C(\beta \mathbb{N})$ of all real-valued continuous functions on the compact Hausdorff space $\beta \mathbb{N}$. Besides, the map $\beta: C^{*}(\mathbb{N}) \rightarrow$ $C(\beta \mathbb{N})$ defined by

$$
\beta(f)=f^{\beta} \quad \text { for all } f \in C^{*}(\mathbb{N})
$$

is an algebra and lattice isomorphism. We also need the elements $\mathbf{1}$ and $\mathbf{j}$ of $C^{*}(\mathbb{N})$ defined by

$$
\mathbf{1}(n)=1 \quad \text { and } \quad \mathbf{j}(n)=n^{-1} \quad \text { for all } n \in \mathbb{N} .
$$

A detailed survey on $\beta \mathbb{N}$ and $C(\beta \mathbb{N})$ can be found in 3 .

Next we assume $E$ to be the set of all $f \in C^{*}(\mathbb{N})$ for which there exist real numbers $\ell_{f}$ and $\alpha$ such that the inequality $\left|f-\ell_{f} \mathbf{1}\right| \leq \alpha \mathbf{j}$ holds in $C^{*}(\mathbb{N})$. It is readily verified that $E$ is a Riesz subspace of $C^{*}(\mathbb{N})$. Moreover, a moment's thought reveals that the Dedekind-completion $E^{\delta}$ of $E$ is precisely $C^{*}(\mathbb{N})$. Furthermore, the formula

$$
\mathbf{j}^{\beta}(r)=0 \quad \text { for all } r \in \beta \mathbb{N}-\mathbb{N}
$$

(see page 102 in [3]) leads directly to

$$
f^{\beta}(r)=\ell_{f} \quad \text { for all } f \in E \text { and } r \in \beta \mathbb{N}-\mathbb{N} .
$$

Put $F=\mathbb{R}$ and let $\Psi: E \times E \rightarrow F$ be the bilinear map defined by

$$
\Psi(f, g)=\ell_{f} \ell_{g} \quad \text { for all } f, g \in E .
$$

It is quite easy to show that $\Psi$ is an orthosymmetric lattice bimorphism. On the other hand, pick $p, q \in \beta \mathbb{N}-\mathbb{N}$ such that $p \neq q$ and let $\Psi^{\delta}: E^{\delta} \times E^{\delta} \rightarrow F$ be the bilinear map defined by

$$
\Psi^{\delta}(f, g)=f^{\beta}(p) g^{\beta}(q) \quad \text { for all } f, g \in E^{\delta} .
$$

It is not hard to see that $\Psi^{\delta}$ is also a lattice bimorphism. Moreover, the formula ( $\star$ ) quickly yields that $\Psi^{\delta}$ is an extension of $\Psi$. However, $\Psi^{\delta}$ is obviously not orthosymmetric, so statement 1.1 is proved.

Now we prove assertion 1.2. To this end, we keep the same notation as previously used to show statement 1.1, and we define a multiplication $*$ in $E$ by

$$
f * g=\Psi(f, g) \mathbf{1} \quad \text { for all } f, g \in E .
$$

Clearly, $*$ is a $d$-multiplication because $\Psi$ is a lattice bimorphism. Similarly, since $\Psi^{\delta}$ is a lattice bimorphism which is an extension of $*$, the multiplication $*^{\delta}$ defined in $E^{\delta}$ by

$$
f *^{\delta} g=\Psi^{\delta}(f, g) \mathbf{1} \quad \text { for all } f, g \in E^{\delta}
$$


is a $d$-multiplication which is an extension of $*$. However, it is an easy exercise to check that $*^{\delta}$ is not associative while $*$ is associative. This completes the proof of assertion 1.2 above.

\section{REFERENCES}

[1] C. D. Aliprantis and O. Burkinshaw, Positive Operators, Academic Press, New York-London, 1985. MR0809372 (87h:47086)

[2] G. Buskes and A. van Rooij, Almost $f$-algebras: structure and the Dedekind completion, Positivity 3 (2000), 233-243. MR 1797126 (2001j:46062)

[3] L. Gillman and M. Jerison, Rings of Continuous Functions, Springer Verlag, Berlin-Heidelberg, 1976. MR0407579(53:11352)

[4] V. Kudláček, On some types of $\ell$-rings, Sb. Vysoké. Učení. Tech. Brno. 1-2 (1962), 179-181. MR0184882(32:2353)

[5] M. A. Toumi, Extensions of orthosymmetric lattice bimorphisms, Proc. Amer. Math. Soc., 134 (2005), 1615-1621. MR2204271

Institut Préparatoire aux Etudes Scientifiques et Techniques, Université 7 novemBre À Carthage, BP51, 2070-La Marsa, Tunisia

E-mail address: karim.boulabiar@ipest.rnu.tn 